\title{
ĐÁNH GIÁ ẢNH HƯởNG CỦA 07 THAM SỐ CHUYỂN ĐỔI TỌA ĐỘ ĐẾN ĐỘ CHÍNH XÁC XÁC ĐỊNH MẠTT QUASIGEOID QUỐC GIA
}

\author{
LƯO'NG THANH THACH \\ Trường Đại học Tài nguyên và Môi trường Hà Nội
}

\section{Tóm tắt:}

Bài báo khoa học này sẽ luận chứng cho việc hiện nay cần phải xây dựng Hệ quy chiếu tọa độ không gian quốc gia mà bản chất là xây dựng mô hình quasigeoid độ chính xác cao. Đồng thời, cũng sẽ chứng minh rằng việc bỏ qua độ cao trắc địa của các điểm trắc địa hạng I, Il quốc gia trùng với các điểm GNSS trong việc xác định 07 tham số chuyển tọa độ từ ellipsoid WGS84 quốc tế về ellipsoid WGS84 quy chiếu không cho phép nhận được độ cao trắc địa đạt độ chính xác cao trong hệ Hệ quy chiếu tọa độ không gian quốc gia (VN2000 $3 D$ ).

\section{1. Đặt vấn đề}

Hiện nay, với sự phát triển mạnh mẽ của công nghệ GNSS (Global Navigation Satellite System) thì việc xây dựng và phát triển Hệ quy chiếu tọa độ không gian quốc gia là hoàn toàn phù hợp với yêu cầu và xu thế phát triển, dựa trên cơ sở ứng dụng các thành tựu Khoa học Công nghệ của trắc địa thế giới. Đó là, Khung quy chiếu Trái Đất quốc tế ITRF (International Terrestrial Reference System) dựa vào công nghệ GNSS, xây dựng các mô hình trọng trường Trái Đất EGM (Earth Gravitational Model) và mô hình địa hình động lực trung bình MDT (Mean Dynamic Topography) nhờ phương pháp đo cao từ vệ tinh (altimtry), xác định mặt geoid toàn cầu trên các biển và đại dương nhằm xác định các tọa độ (không gian, trắc địa, mặt bằng) và độ cao chuẩn (hoặc độ sâu) của điểm cần quan tâm với độ chính xác cao cả trên đất liền và trên biển dựa trên công nghệ GNSS.

Khi xây dựng Hệ tọa độ VN2000, trên cơ sở định vị ellipsoid WGS84 chúng ta dựa trên nguyên tắc định vị ellipsoid sát nhất với mặt geoid cục bộ ở Việt Nam và xác định được các điểm gốc của hệ tọa độ quốc gia cho hệ VN2000. Xét về bản chất thì hệ VN2000 hiện nay là hệ tọa độ mặt phẳng (2D). Để xây dựng hệ quy chiếu tọa độ không gian quốc gia chúng ta phải xác định được độ cao trắc địa với độ chính xác tương đối $\frac{m_{H}}{R}$ ở mức $10^{-9}$ trên toàn bộ lãnh thổ Việt Nam. Do khi xây dựng hệ tọa độ VN2000 chúng ta chưa có mô hình quasigeoid độ chính xác cao, nên 07 tham số hiện nay được công bố trong ngành Trắc địa Bản đồ không cho phép xác định được độ cao trắc địa độ chính xác cao theo yêu cầu của Hệ quy chiếu tọa độ không gian quốc gia. (xem hình 1)

Giả thiết rằng trên tập hợp các điểm trùng trên mặt vật lý Trái Đất, mỗi điểm có tọa độ trắc địa độ chính xác cao $(B, L, H)$ trong hệ tọa độ không gian quốc gia và tọa độ trắc địa độ chính xác cao $(\bar{B}, \bar{L}, \bar{H})$ tương ứng với ellipsoid quy chiếu quốc tế WGS84, thêm vào đó các tọa độ trắc địa $(\bar{B}, \bar{L}, \bar{H})$ được xác định từ các kết quả xử lý các dữ liệu đo GNSS trong ITRF. Trong trường hợp ellipsoid quy chiếu quốc gia và ellipsoid quy chiếu quốc tế cùng là một loại ellipsoid, thêm vào đó ellipsoid quy chiếu quốc gia nhận được từ các kết quả định vị ellipsoid quy chiếu quốc tế cho sát nhất với mặt quasigeoid trên lãnh thổ quốc

Ngày nhận bài: 19/8/2016, ngày chuyển phản biện: 22/8/2016, ngày chấp nhận phản biện: 15/9/2016, ngày chấp nhận đăng: 19/9/2016 


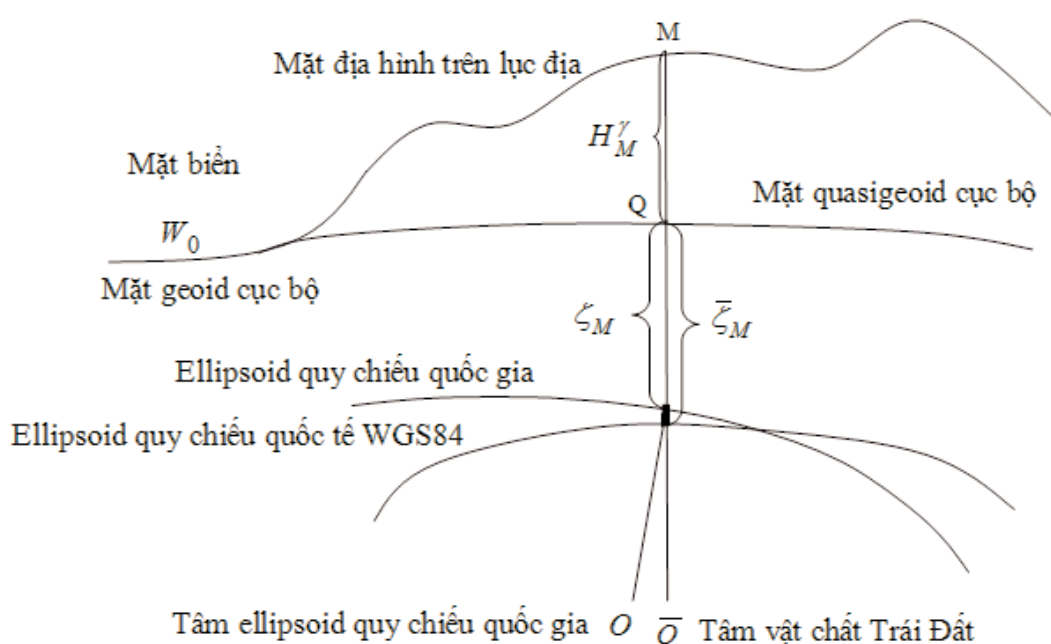

\section{Hinh 1}

gia, mối liên kết giữa các tọa độ trắc địa $(B, L, H)$ và $(\bar{B}, \bar{L}, \bar{H})$ được thể hiện theo các công thức sau (Hà Minh Hòa (2013)):

$$
\begin{gathered}
B=\bar{B}-\frac{1}{\bar{M}+\bar{H}} \cdot\left[\cos \bar{L} \cdot \sin \bar{B} \cdot \tilde{X}_{0}+\sin \bar{B} \cdot \sin \bar{L} \cdot \tilde{Y}_{0}+\cos \bar{B} \cdot \tilde{Z}_{0}\right]- \\
-\left(1+\bar{e}^{2} \cdot \cos 2 \tilde{B}\right) \cdot \sin \tilde{L} \cdot \tilde{\varepsilon}_{x}+\left(1+\bar{e}^{2} \cdot \cos 2 \bar{B}\right) \cdot \cos \bar{L} \cdot \tilde{\varepsilon}_{y}-\bar{e}^{2} \cdot \sin \bar{B} \cdot \cos \bar{B} \cdot \Delta \tilde{m} ; \\
L=\bar{L}+\frac{1}{(\bar{N}+\bar{H}) \cdot \cos \bar{B}} \cdot\left(-\sin \bar{L} \cdot \tilde{X}_{0}+\cos \bar{L} \cdot \tilde{Y}_{0}\right)+\left(1-\bar{e}^{2}\right) \cdot \operatorname{tg} \bar{B} \cdot \cos \bar{L} \cdot \tilde{\varepsilon}_{x}+ \\
+\left(1-\bar{e}^{2}\right) \cdot \operatorname{tg} \bar{B} \cdot \sin \bar{L} \cdot \tilde{\varepsilon}_{y}-\tilde{\varepsilon}_{z} \cdot \\
H=\bar{H}+\cos \bar{B} \cdot \cos \bar{L} \cdot \tilde{X}_{0}+\cos \bar{B} \cdot \sin \bar{L} \cdot \tilde{Y}_{0}+\sin \bar{B} \cdot \tilde{Z}_{0}-\bar{e}^{2} \cdot \bar{N} \cdot \sin \bar{B} \cdot \cos \bar{B} \cdot \sin \bar{L} \cdot \tilde{\varepsilon}_{x}+ \\
+\bar{e}^{2} \cdot \bar{N} \cdot \sin \bar{B} \cdot \cos \bar{B} \cdot \cos \bar{L} \cdot \tilde{\varepsilon}_{y}+\left(\frac{\bar{a}^{2}}{\bar{N}}+\bar{H}\right) \cdot \Delta \tilde{m},
\end{gathered}
$$

ở đây các thành phần $\widetilde{X}_{0}, \widetilde{Y}_{0}, \widetilde{Z}_{0}$ có đơn vị mét $(\mathrm{m})$, các thành phần $\widetilde{\varepsilon}_{x}, \widetilde{\varepsilon}_{y}, \widetilde{\varepsilon}_{z}$ có đơn vị radian, còn thành phần $\Delta \widetilde{m}$ không có thứ nguyên.

Trong trường hợp lý tưởng, khi đã biết độ cao trắc địa $H$ có độ chính xác cao của các điểm trắc địa tương ứng với ellipsoid quy chiếu quốc gia, từ các phương trình (1), (2) đối với các điểm trùng, chúng ta xác định được các giá trị chính xác của các tham số chuyển đổi tọa độ $\widetilde{X}_{0}, \widetilde{Y}_{0}, \widetilde{Z}_{0}, \widetilde{\varepsilon}_{x}, \widetilde{\varepsilon}_{y}, \widetilde{\varepsilon}_{z}, \Delta \widetilde{m}$ từ ITRF về hệ tọa độ không gian quốc gia. Các tham số này đảm bảo sự liên kết giữa ITRF và hệ tọa độ không gian quốc gia trên cơ sở ứng dụng công nghệ GNSS.

Về phần mình, độ cao trắc địa $H$ tương ứng với ellipsoid quy chiếu quốc gia được xác định theo công thức $H=H^{\gamma}+\zeta$, ở đây $H^{\gamma}$. độ cao chuẩn, $\xi$ - dị thường độ cao được xác định từ mô hình quasigeoid quốc gia độ chính xác cao. Để xây dựng được hệ quy chiếu tọa độ không gian quốc gia độ chính xác cao yêu cầu sai số tương đối của các tọa độ không gian $X, Y, Z$ của điểm phải đạt ở mức $10^{-9}$. Để đạt được mức này, trong tài liệu 
(Hà Minh Hòa (2014)) đã chứng minh được rằng mô hình quasigeoid quốc gia phải đạt độ chính xác không thấp hơn $\pm 4,0 \mathrm{~cm}$.

Các hệ quy chiếu tọa độ không gian của một số nước đã được trình bày trong tài liệu (Hà Minh Hòa (2012)). Phân ban Đo đạc trắc địa (The Geodetic Survey Division - GSD) trực thuộc Bộ Tài nguyên của Canada (Natural Resources Canada - NRCan) đã triển khai dự án hiện đại hóa hệ độ cao Canada từ năm 2002 đến năm 2013. Hệ độ cao hiện đại CGVD2013 của Canada (the Canadian Geodetic Vertical Datum of 2013) được xây dựng dựa trên mặt geoid toàn cầu. Mô hình geoid CGG2013 của Canada (Canadian Gravimetric Geoid of 2013) có độ chính xác cao hơn $\pm 3.0 \mathrm{~cm}$. Hệ độ cao của Vương quốc Anh có tên ODN (Ordnance Datum Newlyn) được xây dựng dựa trên mô hình geoid trọng lực OSGM02 có độ chính xác cao hơn $\pm 2.0 \mathrm{~cm}$ trong lãnh thổ Vương quốc Anh và $\pm 4.0 \mathrm{~cm}$ ở các khu vực khác. Đến năm 2002, các mạng lưới tam giác OSTN (Ordance Survey Triangulation Network) được nối với hệ quy chiếu Trái Đất Châu Âu ETRS89. Kết quả đã xây dựng được hệ tọa độ không gian địa tâm OS Net (Ordnance Survey Network) của Vương quốc Anh được liên kết với ETRS89.

Hiện nay, Liên bang Nga mới xác định được dị thường độ cao ở mức $0.2 \mathrm{~m}-0.3 \mathrm{~m}$. Chính vì lý do này mà ở Liên bang Nga chưa xây dựng được hệ tọa độ không gian quốc gia. Ở Trung Quốc cũng tương tự, năm 1999 Trung Quốc đã xây dựng mô hình geoid DQM99A trên cơ sở sử dụng các dữ liệu trọng lực trong các ô chuẩn 5'x5' và 63 điểm GPS/thủy chuẩn cách nhau $100 \mathrm{~km}$ có liên kết với 5 trạm nghiệm triều. Kết quả đo GPS được liên kết với mạng lưới IGS. Năm 2001 Trung Quốc đã phát triển thêm 950 điểm GPS/thủy chuẩn để xây dựng mô hình geoid với độ chính xác $0.5 \mathrm{~m}$. Do độ chính xác của mô hình geoid của Trung Quốc chưa cao, nên Trung Quốc chưa xây dựng được hệ tọa độ không gian quốc gia. Ở Australia, Hệ tọa độ mặt bằng của Australia là AGD66 (Australia Geodetic Datum 1966) có điểm gốc là Johnston dựa trên ellipsoid (còn gọi là quả cầu quốc gia Australia). Năm 1982 tiến hành bình sai mạng lưới trắc địa quốc gia đã xây dựng được hệ tọa độ không gian địa tâm AGD84 (The Geocentric Datum of Australia - GDA). Gốc của AGD84 trùng với tâm vật chất của Trái Đất. Do đó AGD84 là hệ tọa độ địa tâm. Ở Papua New Guinea, Hệ tọa độ không gian địa tâm PND94 (Papua New Guinea's Geodetic Datum 1994) của Papua New Guinea được xây dựng cùng với hệ tọa độ không gian AGD94 của Australia dựa trên ITRF92 với thời điểm chuẩn 1994. Ở New Zealand đã đề xuất xây dựng hệ tọa độ địa tâm mới cho New Zealand dựa trên ITRF2000 nhằm hoàn thiện hệ thống tọa độ cho New Zealand và xác định chuyển dịch của vỏ Trái Đất.

Trong thực tế ở Việt Nam hiện nay chưa có mô hình quasigeoid quốc gia với độ chính xác nêu ở trên. Do hệ tọa độ VN2000 là hệ tọa độ phẳng, nên để xác định 07 tham số chuyển đổi tọa độ theo mô hình Bursa - Wolf, đã coi các độ cao trắc địa $H$ bằng 0 , tức sử dụng các điểm trùng đã được quy chiếu lên mặt ellipsoid. Trong trường hợp này chúng ta sử dụng công thức (1) để xác định 07 tham số chuyển đổi tọa độ gần đúng $X_{0}, Y_{0}, Z_{0}$, $\varepsilon_{x}, \varepsilon_{y}, \varepsilon_{z}, \Delta m$. Lúc này công thức (1) được viết lại dưới dạng:

$$
\left.\begin{array}{l}
B=\bar{B}-\frac{1}{\bar{M}}\left[\cos \bar{L} \cdot \sin \bar{B} \cdot \widetilde{X}_{0}+\sin \bar{B} \cdot \sin \bar{L} \cdot \widetilde{Y}_{0}+\cos \bar{B} \cdot \widetilde{Z}_{0}\right]- \\
-\left(1+\bar{e}^{2} \cdot \cos 2 \bar{B}\right) \sin \bar{L} \cdot \widetilde{\varepsilon}_{X}+\left(1+\bar{e}^{2} \cdot \cos 2 \bar{B}\right) \cos \bar{L} \cdot \widetilde{\varepsilon}_{Y}-\bar{e}^{2} \cdot \sin \bar{B} \cdot \cos \bar{B} \cdot \Delta \widetilde{m} \\
L=\bar{L}+\frac{1}{\bar{N} \cdot \cos \bar{B}}\left(-\cdot \sin \bar{L} \cdot \widetilde{X}_{0}+\cos \bar{L} \cdot \widetilde{Y}_{0}\right)+\left(1-\bar{e}^{2}\right) \operatorname{tg} \bar{B} \cdot \cos \bar{L} \cdot \widetilde{\varepsilon}_{X}+ \\
+\left(1-\bar{e}^{2}\right) \operatorname{tg} \bar{B} \cdot \sin \bar{L} \cdot \widetilde{\varepsilon}_{Y}-\widetilde{\varepsilon}_{Z}
\end{array}\right\}
$$


Cách làm nêu trên đảm bảo việc chuyển đổi độ chính xác cao các tọa độ trắc địa $\bar{B}, \bar{L}$ từ ellipsoid quy chiếu quốc tế WGS84 thành các tọa độ trắc địa $B, L$ tương ứng với ellipsoid quy chiếu quốc gia, nhưng không đảm bảo việc chuyển đổi độ chính xác cao giá trị độ cao trắc địa $\overline{H K}$ từ ellipsoid quy chiếu quốc tế WGS84 thành độ cao trắc địa $H$ tương ứng với ellipsoid quy chiếu quốc gia.

Bài báo khoa học này sẽ đánh giá độ sai lệch của các giá trị độ cao trắc địa $H$ khi chuyển đổi các giá trị độ cao trắc địa $\overline{I_{I}}$ từ ellipsoid quy chiếu quốc tế WGS84 về hệ tọa độ không gian quốc gia theo 07 tham số chuyển đổi tọa độ gần đúng $X_{0}, Y_{0}, Z_{0}, \varepsilon_{x}, \varepsilon_{y}, \varepsilon_{z}, \Delta m$.

\section{Giải quyết vấn đề}

\subsection{Cơ sở lý thuyết}

Do các trục tọa độ $\mathrm{OX}, \mathrm{OY}, \mathrm{OZ}$ liên quan chặt chẽ với ellipsoid, nên đối với các điểm trùng đã được quy chiếu lên mặt ellipsoid, việc xác định các tham số $\varepsilon_{x}, \varepsilon_{y}, \varepsilon_{z}, \Delta m$ được coi là tin cậy. Tuy nhiên, đối với hệ quy chiếu tọa độ không gian quốc gia chúng ta phải đảm bảo để thông qua 07 tham số của mô hình Bursa - Wolf nhận được các tọa độ trắc địa độ chính xác cao $(B, L, H)$ của điểm cần quan tâm trên mặt vật lý Trái Đất từ phép chuyển các tọa độ trắc địa $(\bar{B}, \bar{L}, \bar{H})$ được xác định từ các kết quả xử lý dữ liệu đo GNSS trong ITRF tương ứng với ellipsoid quy chiếu quốc tế WGS84. Giả thiết đối với hệ quy chiếu tọa độ không gian quốc gia VN2000 3D, tọa độ không gian của điểm được xác định theo công thức:

$$
\begin{aligned}
& X=(N+H) \cdot \cos B \cdot \cos L, \\
& Y=(N+H) \cdot \cos B \cdot \sin L, \\
& Z=\left[N \cdot\left(1-e^{2}\right)+H\right] \cdot \sin B,
\end{aligned}
$$

ở đây bán kính cong trên mặt phẳng thẳng đứng thứ nhất $N=a \cdot\left(1-e^{2} \cdot \sin ^{2} B\right)^{-1 / 2}$.

Từ (4) không khó khăn để nhận thấy rằng với sai số trung phương của độ cao trắc địa $H$, đối với bán kính - vectơ $\rho=\sqrt{X^{2}+Y^{2}+Z^{2}}$, sai số trung phương của nó $m_{p}=m_{H}$. Như vậy khi sử dụng các phương trình (3) để tính toán 07 tham số chuyển đổi tọa độ gần đúng $X_{0}, Y_{0}, Z_{0}, \varepsilon_{x}, \varepsilon_{y}, \varepsilon_{z}, \Delta m$, độ cao trắc địa $H$ sẽ chứa sai số trung phương $m_{H}$ và điều này gây ra các sai số chủ yếu trong các tham số $X_{0}, Y_{0}, Z_{0}$, chúng ta sẽ đánh giá mức của các sai số này.

Khi ký hiệu $\widetilde{X}_{0}=X_{0}+\delta X_{0}, \widetilde{Y}_{0}=Y_{0}+\delta Y_{0}, \widetilde{Z}_{0}=Z_{0}+\delta Z_{0}$, từ (2) chúng ta có:

ở đây

$$
H=\hat{\bar{H}}+\cos \bar{B} \cdot \cos \bar{L} \cdot \delta X_{0}+\cos \bar{B} \cdot \sin \bar{L} \cdot \delta Y_{0}+\sin \bar{B} \cdot \delta Z_{0},
$$

$$
\begin{aligned}
& \hat{\bar{H}}=\bar{H}+\cos \bar{B} \cdot \cos \bar{L} \cdot X_{0}{ }^{+} \\
& +\cos \bar{B} \cdot \sin \bar{L} \cdot Y_{0}+\sin \bar{B} \cdot Z_{0}-\bar{e}^{2} \cdot \bar{N} \cdot \sin \bar{B} \cdot \cos \bar{B} \cdot \sin \bar{L} \cdot \varepsilon_{x}+ \\
& +\bar{e}^{2} \cdot \bar{N} \cdot \sin \bar{B} \cdot \cos \bar{B} \cdot \cos \bar{L} \cdot \varepsilon_{y}+\left(\frac{\bar{a}^{2}}{\bar{N}}+\bar{H}\right) \cdot \Delta m .
\end{aligned}
$$

Khi ký hiệu $H^{\gamma}$ là độ cao chuẩn của điểm tương ứng với mặt geoid cục bộ Hòn Dấu, thì công thức (5) được viết lại dưới dạng:

$$
\zeta=\hat{\bar{\zeta}}+\cos \bar{B} \cdot \cos \bar{L} \cdot \delta X_{0}+\cos \bar{B} \cdot \sin \bar{L} \cdot \delta Y_{0}+\sin \bar{B} \cdot \delta Z_{0},
$$


Ở đây $\zeta=H-Y^{\prime}$ - dị thường độ cao được xác định từ mô hình quasigeoid quốc gia độ chính xác cao, còn từ công thức (6) chúng ta xác định được dị thường độ cao:

$$
\begin{aligned}
& \hat{\bar{\zeta}}=\hat{\bar{H}}-H^{\gamma}=\bar{H}-H^{\gamma}+\cos \bar{B} \cdot \cos \bar{L} \cdot X_{0}{ }^{+} \\
& +\cos \bar{B} \cdot \sin \bar{L} \cdot Y_{0}+\sin \bar{B} \cdot Z_{0}-\bar{e}^{2} \cdot \bar{N} \cdot \sin \bar{B} \cdot \cos \bar{B} \cdot \sin \bar{L} \cdot \varepsilon_{x}+ \\
& +\bar{e}^{2} \cdot \bar{N} \cdot \sin \bar{B} \cdot \cos \bar{B} \cdot \cos \bar{L} \cdot \varepsilon_{y}+\left(\frac{\bar{a}^{2}}{\bar{N}}+\bar{H}\right) \cdot \Delta m= \\
& =\bar{\zeta}+\cos \bar{B} \cdot \cos \bar{L} \cdot X_{0}+\cos \bar{B} \cdot \sin \bar{L} \cdot Y_{0}+\sin \bar{B} \cdot Z_{0}- \\
& -\bar{e}^{2} \cdot \bar{N} \cdot \sin \bar{B} \cdot \cos \bar{B} \cdot \sin \bar{L} \cdot \varepsilon_{x}+\bar{e}^{2} \cdot \bar{N} \cdot \sin \bar{B} \cdot \cos \bar{B} \cdot \cos \bar{L} \cdot \varepsilon_{y}+\left(\frac{\bar{a}^{2}}{\bar{N}}+\bar{H}\right) \cdot \Delta m .
\end{aligned}
$$

Ở đây: $\bar{\zeta}$ - là dị thường độ cao được tính từ mô hình VIGAC2014 tương ứng với ellipsoid WGS84 chưa định vị lại ở Việt Nam; $X_{0}, Y_{0}, Z_{0}, \varepsilon_{x}, \varepsilon_{y}, \varepsilon_{z}, \Delta m$ - 07 tham số định vị ellipsoid WGS84 toàn cầu về ellipsoid quy chiếu quốc gia. Chúng ta tính cho hai trường hợp, (1) là 07 tham số có giá trị được cho trong tài liệu (Bộ Tài nguyên và Môi trường (2007)) và (2) là 07 tham số có giá trị được Cục Bản đồ - Bộ Tổng tham mưu cung cấp.

Lúc này từ (7) chúng ta có phương trình số cải chính:

$$
V_{\zeta}=\cos \bar{B} \cdot \cos \bar{L} \cdot \delta X_{0}+\cos \bar{B} \cdot \sin \bar{L} \cdot \delta Y_{0}+\sin \bar{B} \cdot \delta Z_{0}+(\hat{\bar{\zeta}}-\zeta)
$$

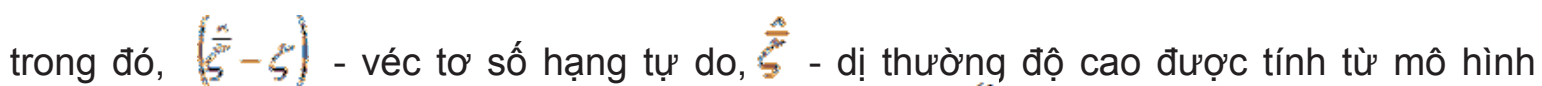
VIGAC2014 tương ứng với mặt geoid cục bộ Hòn Dấu, $\zeta$ - là dị thường độ cao được lấy trong bảng IV.3.2 của tài liệu (Tổng cục Địa chính (1999)). Giải (9) theo nguyên lý số bình phương nhỏ nhất chúng ta sẽ tìm được $\delta X_{0}, \delta Y_{0}, \delta Z_{0}$.

Sau khi tìm được $\delta X_{0}, \delta Y_{0}, \delta Z_{0}$ ở bước trên, chúng ta thay $\delta X_{0}, \delta Y_{0}, \delta Z_{0}$ trở lại vào (9) để xác định các giá trị ${ }^{P}$ - là giá trị thể hiện cho độ lệch khi định vị ellipsoid mà bỏ qua độ cao trắc địa, với lưu ý rằng được tính theo công thức (8).

\subsection{Tính toán thực nghiệm}

Trong phần tính toán thực nghiệm này, chúng ta sử dụng 09 điểm cấp 0 trong tổng số 25 điểm cơ sở định vị được cho trong bảng IV.3.2 của tài liệu (Tổng cục Địa chính (1999)). Dưới đây là bảng thống kê tọa độ trắc địa $B, L$; dị thường độ cao $\frac{\tilde{m}}{\bar{\zeta}}$ được tính từ mô hình VIGAC2014 tương ứng với mặt geoid cục bộ Hòn Dấu; dị thường độ cao $\bar{\zeta}$ được tính từ mô hình VIGAC2014 tương ứng với ellipsoid WGS84 chưa định vị tại Việt Nam và dị thường độ cao $\zeta$ sau khi định vị ellipsoid quy chiếu tại Việt Nam được trích trong trong bảng IV.3.2 của tài liệu (Tổng cục Địa chính (1999)). (xem bảng 1)

Dựa vào số liệu đã cho ở bảng 1 , tiến hành lập phương trình số hiệu chỉnh cho 09 điểm theo (9), chúng ta có ma trận hệ số của hệ phương trình (9) và véc tơ số hạng tự do như sau: 
Bảng 1: Các điểm cơ sở định vị cấp 0

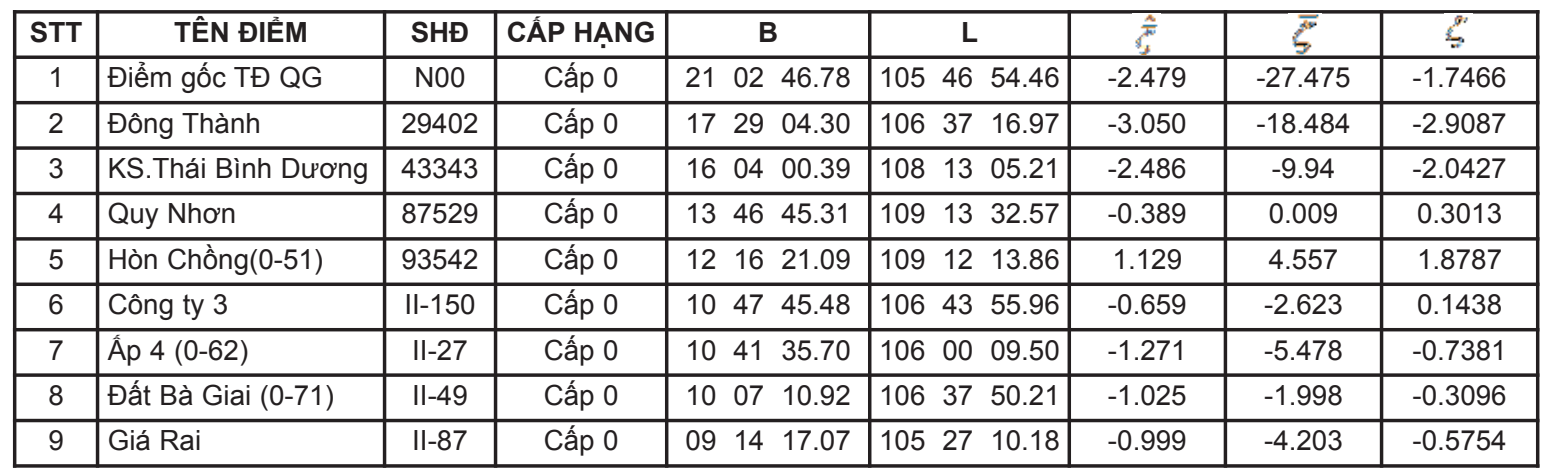

$$
A=\left[\begin{array}{ccc}
-0.25383 & 0.89811 & 0.35912 \\
-0.27283 & 0.91394 & 0.30045 \\
-0.30042 & 0.91277 & 0.27676 \\
-0.31981 & 0.91705 & 0.23818 \\
-0.32141 & 0.92277 & 0.21256 \\
-0.28280 & 0.94071 & 0.18731 \\
-0.27089 & 0.94456 & 0.18555 \\
-0.28175 & 0.94326 & 0.17571 \\
-0.26299 & 0.95135 & 0.16054
\end{array}\right] \quad(\hat{\bar{\zeta}}-\zeta)=\left(\begin{array}{c}
-0.73240 \\
-0.14130 \\
-0.44330 \\
-0.69030 \\
-0.65970 \\
-0.80280 \\
-0.53290 \\
-0.71540 \\
-0.42360
\end{array}\right)
$$

Giải (9) theo nguyên lý số bình phương nhỏ nhất, chúng ta được nghiệm:

$$
\delta X_{0}=-1.7365(\mathrm{~m}) ; \delta Y_{0}=0.1947(\mathrm{~m}) ; \delta Z_{0}=-0.4491(\mathrm{~m})
$$

Tiếp theo, giải (8), (9) theo hai trường hợp là sử dụng 07 tham số định vị được Bộ Tài nguyên và Môi trường công bố trong tài liệu (Bộ Tài nguyên và Môi trường (2007)) và 07 tham số định vị được Cục Bản đồ - Bộ Tổng tham mưu cung cấp, chúng ta xác định được 09 giá trị $V_{\zeta}$ thể hiện cho độ lệch khi định vị ellipsoid mà bỏ qua độ cao trắc địa được thống kê trong bảng. (xem bảng 2)

Theo bảng 2, giá trị $V_{\zeta}$ tại điểm 29402 là lớn nhất trong cả hai trường hợp, nên lúc này chúng ta có sai số trung phương tương đối $\frac{m_{H}}{R}=\frac{m_{\zeta}}{R}$ (với $R=6356752,314 \mathrm{~m}$ - là bán kính trung bình của Trái Đất) lần lượt trong hai trường hợp theo các tham số của Bộ Tài nguyên và Môi trường, Cục Bản đồ - Bộ Tổng Tham mưu cung cấp là: $5.95 \times 10^{-8}$ và $5.44 \times 10^{-8}$. Trong khi đó, để xây dựng Hệ quy chiếu tọa độ không gian quốc gia phải đảm bảo sai số tương đối của các thành phần tọa độ không gian ở mức 1×10-9 (Hà Minh Hòa (2012), trang 89 và Hà Minh Hòa (2013b), trang 213).

Như vậy, chúng ta có thể kết luận rằng, độ chính xác độ cao trắc địa của điểm thiên văn trắc địa quốc gia trong hệ VN2000 nhận được từ kết quả định vị ellipsoid là không cao, tức sai số trung phương tương đối của độ cao trắc địa của các điểm thiên văn trắc địa chỉ ở mức $5 \times 10^{-8}$ và thấp hơn nhiều so với độ chính xác của độ cao trắc địa trong ITRF (mức $\left.1 \times 10^{-9}\right)$. 
Bảng 2: Giá trị độ lệch khi định vị ellipsoid bỏ qua độ cao trắc địa

\begin{tabular}{|c|c|c|c|c|}
\hline STT & Số hiệu điểm & Độ lệch $V_{\zeta}$ & $\begin{array}{c}\text { Bộ TNMT } \\
\text { (m) }\end{array}$ & $\begin{array}{c}\text { BTTM } \\
\text { (m) }\end{array}$ \\
\hline 1 & N00 & $V_{\zeta_{N 00}}$ & -0.275 & -0.295 \\
\hline 2 & 29402 & $V_{\zeta_{29402}}$ & 0.378 & 0.346 \\
\hline 3 & 43343 & $V_{\zeta_{43343}}$ & 0.132 & 0.078 \\
\hline 4 & 87529 & $V_{\zeta 87529}$ & -0.063 & -0.125 \\
\hline 5 & 93542 & $V_{\zeta 93542}$ & -0.015 & -0.068 \\
\hline 6 & II-150 & $V_{\zeta_{I I-150}}$ & -0.212 & -0.215 \\
\hline 7 & II-27 & $V_{\zeta_{I I-27}}$ & 0.036 & 0.046 \\
\hline 8 & II-49 & $V_{\zeta_{1}}$ & -0.120 & -0.117 \\
\hline 9 & II-87 & $V_{\zeta_{I I-87}}$ & 0.143 & 0.174 \\
\hline & & & 0.321 & 0.326 \\
\hline \multicolumn{2}{|c|}{$\begin{array}{l}\text { Sai số trung phương } \\
(\mathrm{m})\end{array}$} & $m_{0}= \pm \sqrt{\frac{1 \zeta}{n}}$ & \pm 0.189 & \pm 0.190 \\
\hline
\end{tabular}

Mặt khác, giả thiết rằng 09 điểm cơ sở cấp 0 trên không có sai số, chúng ta có độ chính xác xác định độ lệch dị thường độ cao $V_{\zeta_{i}}$ được thống kê trong bảng 2 trong hai trường hợp lần lượt là $\pm 0.189 \mathrm{~m}$ và $\pm 0.190 \mathrm{~m}$. Trong khi đó, (theo tài liệu Hà Minh Hòa (2013b), trang 215), để xây dựng Hệ quy chiếu tọa độ không gian quốc gia, chúng ta phải xác định được dị thường độ cao ś của các điểm cơ sở của khung quy chiếu quốc gia với độ chính xác không thấp hơn $\pm 4.0 \mathrm{~cm}$ trong trường hợp đo nối thủy chuẩn hạng I từ các điểm độ cao hạng I quốc gia vào các điểm cơ sở.

\section{Kết luận}

Việt Nam hiện nay đang trong quá trình hoàn thiện Hệ độ cao gắn liền với việc xây dựng Hệ quy chiếu tọa độ không gian quốc gia nhưng chúng ta chưa có mô hình quasigeoid độ 
chính xác cao, nên 07 tham số định vị đã công bố trong ngành Trắc địa Bản đồ được xác định với trường hợp độ cao trắc địa bằng 0 . Do đó, 07 tham số này sẽ gây ra sai số lớn trong độ cao trắc địa khi chuyển các trị đo GNSS từ hệ WGS84 về hệ VN2000 (sai lệch cỡ $\mathrm{dm}$, như theo tính toán thực nghiệm là xấp xỉ $2 \mathrm{dm}$ ). Với sai số này thì không thể đáp ứng được yêu cầu đối với hệ quy chiếu tọa độ không gian quốc gia. Do vậy, vấn đề đặt ra đối với ngành Trắc địa Bản đồ là bắt buộc phải xây dựng mô hình quasigeoid độ chính xác cao (cao hơn $\pm 4.0 \mathrm{~cm}$ ). Đây là điều kiện bắt buộc để xây dựng Hệ quy chiếu tọa độ không gian quốc gia trên lãnh thổ Việt Nam.

Muốn xây dựng hệ quy chiếu tọa độ không gian quốc gia Việt Nam độ chính xác cao, phải xây dựng mô hình quasigeoid độ chính xác cao để đảm bảo độ cao trắc địa được xác định với sai số trung phương tương đối ở mức $10^{-9}$. Điều này không chỉ đảm bảo xác định 07 tham số chính xác theo các phương trình (1), (2) mà còn đảm bảo gắn kết chặt chẽ giữa hệ tọa độ không gian quốc gia và ITRF.

Muốn làm được như vậy phải sử dụng đồng bộ các dữ liệu GNSS, các dữ liệu độ cao hạng I, II quốc gia, các dữ liệu của các mô hình quasigeoid/geoid toàn cầu độ chính xác cao (như EGM2008), sử dụng các dữ liệu trọng lực ở Việt Nam, .v.v...

Theo kết quả nghiên cứu trong tài liệu (Vũ Xuân Cường (2015)), trong thực tế các mốc độ cao quốc gia bị biến động nhiều do các tác động của tự nhiên và nhân sinh. Vì vậy, muốn sử dụng hệ thống độ cao quốc gia để hoàn thiện mô hình quasigeoid độ chính xác cao, ngoài việc phát triển dữ liệu trọng lực ở Việt Nam để chính xác hóa các hệ số khai triển điều hòa của các mô hình toàn cầu cho phù hợp với lãnh thổ Việt Nam, cần phải hiện đại hóa hệ độ cao quốc gia. $O$

\section{Tài liệu tham khảo}

[1]. Bộ Tài nguyên và Môi trường. (2007). Hướng dẫn sử dụng các tham số tính chuyển từ Hệ tọa độ quốc tế WGS-84 sang Hệ tọa độ quốc gia VN-2000 và ngược lại. Hà Nội, 26/10/2007.

[2]. Hà Minh Hòa (2008). Điểm gốc hệ tọa độ quốc gia - Vai trò và ý nghĩa của nó trong quá trình phát triển hệ tọa độ quốc gia. Tạp chí Địa chính. Số 1 -2/2008, trg. 6 - 13 .

[3]. Hà Minh Hòa (2012). Nghiên cứu cơ sở khoa học của việc hoàn thiện hệ độ cao gắn liền với việc xây dựng hệ tọa độ động lực quốc gia. Báo cáo tổng kết khoa học và kỹ thuật của đề tài nghiên cứu khoa học cấp Bộ. Bộ Tài Nguyên và Môi Trường. Hà Nội - 12/2012.

[4]. Hà Minh Hòa. (2013). Phương pháp chuyển đổi tọa độ giữa các hệ tọa độ. NXB Khoa học và Kỹ thuật, 140 trg. Hà Nội 2013. 
[5]. Hà Minh Hòa (2014). Phương pháp xử lý toán học các mạng lưới trắc địa Quốc gia. NXB Khoa học và Kỹ thuật, 244 trg. Hà Nội 2013.

[6]. Hà Minh Hòa (2016a). Nghiên cứu đánh giá các mặt chuẩn mực nước biển (mặt "0" độ sâu, trung bình và cao nhất) theo các phương pháp trắc địa, hải văn và kiến tạo hiện đại phục vụ xây dựng các công trình và quy hoạch đới bờ Việt Nam trong xu thế biến đổi khí hậu. Báo cáo tổng hợp kết quả khoa học công nghệ đề tài. Chương trình khoa học và công nghệ trọng điểm cấp Nhà nước mã số : KC.09/11-15, Bộ Khoa học và Công nghệ, năm 2016.

[7]. Hà Minh Hòa (2016b). Hướng dẫn sử dụng phần mềm VIGAC2014. Hà Nội, 5/2016.

[8]. Marc Véronneau (2015). Canada's Geodetic Reference Frames: 'Geometric and Vertical. 2015 Geospatial Summit'. Natural Resources Canada.

[9]. Military topographic department of the general staff of armed forces of the Russian federation (2014). "Parametry zenli 1990" (PZ - 90.11). Referencen Document. Moscow 2014.

[10]. Tổng cục Địa chính (1999). Báo cáo khoa học Xây dựng hệ quy chiếu và hệ tọa độ quốc gia. Hà Nội.

[11]. Vũ Xuân Cường (2015). Đề xuất quy trình chọn điểm chôn mốc độ cao quốc gia mới cho khu vực phía Nam. Tạp chí Khoa học Đo đạc và Bản đồ. Số 24 - 6/ 2015, trg. 30 $-3.0$

\section{Summary}

\section{Evaluation of the influences of seven coordinate conversion parameters on the accuracy of the national quasigeoid determination}

\section{Luong Thanh Thach, Hanoi University of Natural Resources and Environment}

This Journal will present a justification for the necesity to build the national space coordinates reference system, which is actually modeling the quasigeoid with high accuracy. At the same time, it will also prove that the obmission of geodetic height of the level I, II national geodetic points coincides with the GNSS points in determining 07 parameters of transfering the coordinates from international WGS84 ellipsoid to national WGS84 ellipsoid, not allowing to receive geodetic height with high accuracy in the national space coordinates reference system (VN20003D). O 